\title{
Obtaining Nanowires under Conditions of Electrodischarge Treatment
}

\author{
Dikusar Alexandr \\ Shevchenko State University, Tiraspol, \\ Pridnestrovie Institute of Applied Physics, \\ Academy of Science of Moldova, Chishinau, \\ Republic of Moldova
}

\section{Introduction}

At present various methods for obtaining nanowires and nanotubes are known using different materials. Nevertheless, the list of these methods grows constantly. This may be accounted for by the fact that, on the one hand, new methods for developing nanomaterials appear using both the technology of bottom-up and top-down. On the other hand, it becomes clear that nanowires and nanotubes can be manufactured using the methods and technologies that are known for a long time under certain conditions.

One such method is the electrodischarge treatment that is the basis for the electrodischarge machining, the method that was proposed more than 50 years ago by the spouses B.R. Lazarenko and N.I. Lazarenko .

This work describes the peculiarities of application of the electrodischarge machiningelectrodischarge doping. The conditions for manufacturing nanowires are described along with certain mechanical properties of the surfaces that are developed by introducing the nanowires into the surface layer composition.

\section{Electrodischarge machining (EDM) and its technological use}

When a certain value of a critical voltage $U_{c r}$ is applied across the interelectrode gap (IEG) that consists of two electrodes and is filled with a dielectric liquid (kerosene or deionized water) the electrical breakdown of the gap (i.e., the formation of the electroconducting region in this medium) is registered. The order of lifetime in this region is $\sim 10^{-7} \mathrm{~s}$ (Fig. 1)

$$
\mathrm{U}_{\mathrm{cr}}=l \mathrm{E}_{\mathrm{cr}},
$$

where $E_{\mathrm{cr}}$ is the critical value of the field intensity that induces the gap breakdown (a discharge); $l$ is the distance between the electrodes.

Since both electrodes in the considered situation have a natural roughness, $E_{c r}$ will be reached firstly at the points with a minimum interelectrode distance $l_{\min }$.

The electron flow that forms on the cathode, evaporates and ionizes the liquid due to its motion to the counter electrode. By the moment the electron avalanche reaches the anode, this flow turns out to be separated from the environment (the liquid) by the vapor-gas- 
plasma cover. After the IEG breakdown the discharge channel tends to be wider and a shock wave is followed by forcing out the liquid in the radial direction with respect to the discharge channel axis. High pressure forms at a front of the shock wave. A certain part of the electric energy introduced into the IEG is transformed by the shock wave into the mechanical work of compression in the working medium. The channel radius is generally less than $10^{-1} \mathrm{~mm}$, the duration of this part of the discharge is short, i.e., within a few microseconds the front moves away for such distances that the energy gain becomes insufficient to ionize the substance.

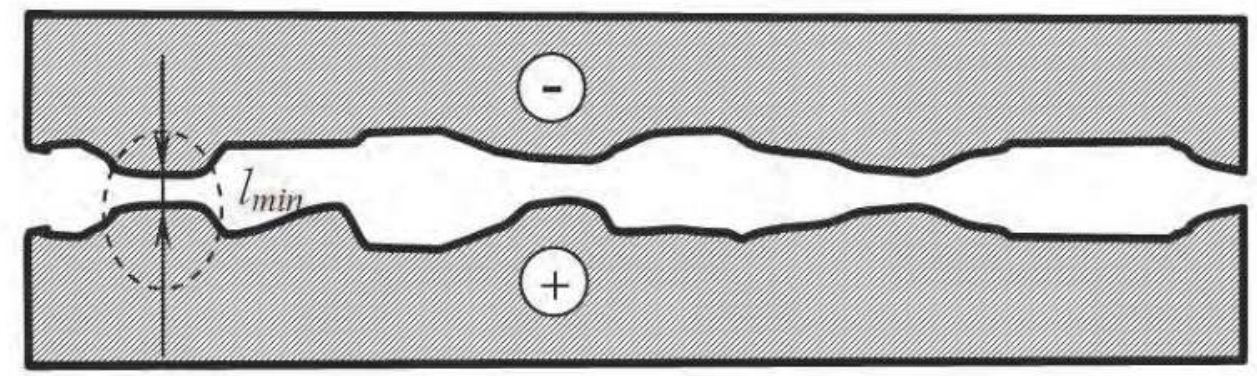

Fig. 1. Scheme of the electric discharge formation.

The EDM is usually characterized by the pulsed supply of the voltage, and during a single pulse the applied voltage changes from $\sim 200$ to $23-25 \mathrm{~V}$, while the lifetime of the plasma channel that arises is up to $200 \mu \mathrm{s}$. Moreover, during the time of $10^{-6}-10^{-7} \mathrm{~s}$ an abrupt increase in the electric current occurs, and the expanding front of the discharge wave increases the radius of the discharge channel. The energy densities within a single pulse reach $3 \mathrm{~J} / \mathrm{mm}^{2}$. The situation after the breakdown is referred to as a spark form of a discharge. It is characterized by the times of $\sim 10^{-8}-10^{-7} \mathrm{~s}$, the current densities of $10^{6}-10^{7} \mathrm{~A} / \mathrm{cm}^{2}$ and temperatures of $10^{4}-10^{5} \mathrm{~K}$. The high local temperature in the discharge channel ensures a possibility of phase transitions across both electrodes, since the obtained temperatures may exceed not only melting but also boiling temperatures.

The removal of the material from the surfaces of both electrodes results from the spark discharge. It concerns the anode in a greater degree, since the cathode melting, as a rule, takes more time versus that of the anode melting. The reason for this is that the electrons have a higher mobility and, hence, reaching a high temperature followed by melting and evaporation of part of the surface starts from the initial period of a pulse (during a few microseconds). The less mobile ions, unlike electrons, ensure the phase transitions across the cathode with a time delay.

After-the-breakdown stage is characterized by a sharp collapse of the discharge plasma channel and the formation of a gaseous bubble. The melted parts of the surface are removed from the surface of the electrodes and are transferred (in a solid state form) into the liquid. The radius of the formed cavities depends on the energy of a single pulse and ranges from $1 \mu \mathrm{m}$ to $\sim 100 \mu \mathrm{m}$.

The rate of erosion is determined by the volume of a sum of cavities that are removed from the surface per the unit of time. The volume of a single dimple determines also the roughness of the surface after the treatment, which is formed by the overlap of single dimples. The erosion 
causes an increase in the value of the local IEG (Fig. 1) and a transition of the discharge to another IEG point. In other words, the considered form of a discharge is a certain form of a non-stationary discharge and a local melting (and evaporation) of the electrode material is the basis of the electroerosion method of treatment that is most popular today.

The electroerosion treatment is performed under the pulse conditions. A pulse generator supplies the currents with several tens of amperes at a regular frequency in the range from the units to hundreds of $\mathrm{kHz}$. An ejection of the melt from the zone of a spark discharge can occur both at the moment of the pulse supply and after its termination. Various hypotheses exist to account for the mechanism of the material removal from the zone of treatment, namely:

- A single ejection of the melt from the erosion dimple at a minimum pressure in the vapor-gas bubble that resulted from a single discharge;

- An ejection of the melt affected by the ponderomotive forces (a current pulse generates a strong magnetic field); the interaction between the vortex current and the magnetic field (that induced the latter) leads to arising the electrodynamic forces;

- Due to the presence of the pressure of the vapors of the materials evaporated from the surface;

- The emission of the products of destruction during the electroerosion treatment of brittle materials that results from the nonuniform thermal expansion of the material and arising thermal strains in the latter.

It is obvious that the EDM real process occurs under the conditions of a simultaneous effect of several factors that determine both the destruction and the emission of the destruction products from the discharge zone.

At present, the EDM serves the following purposes: a 3D copying, producing holes (including those of irregular shapes), treatment and a complicated-profile cutting using an electrode-wire, and the combined treatment (electroerosion polishing), etc. One form of the EDM is an electrospark doping (ESD) which is a process based on a polar transfer of the anode material onto the cathode under the conditions of a spark discharge in a gaseous phase.

\section{ESD - pulsed air arc deposition}

Under the ESD conditions, both electrodes are eroded during the discharge pulse. For the case of the ESD, the anode is less than a cathode, and the cathode surface is treated by the anode (i.e., the anode material is transferred onto the cathode surface).

The basis of this process, just as that of the EDM, is the local melting (evaporation) of the anode material. However, since the transfer occurs in air medium, the surface coating always contains oxides, nitrides, carbides, etc.

The advantages of the EDS are the following:

- The possibility of using different materials in order to change the properties of a surface layer and participation of the interelectrode medium allow one to extensively modify the surface properties and to obtain hard, wear resistant, temperature-resistant, corrosion-resistant, antifriction, and decorative coatings, along with the repair and reconditioning the auto-workpieces;

- The method is simple for implementation and is comparatively cheap;

- $\quad$ The deposited layer has a strong cohesion with the substrate;

- The preliminary surface preparation is unnecessary. 
At the same time, a relatively high roughness of the manufactured surface and the restrictions that result from the impossibility to produce fairly thick layers, restrict its more extended application.

In order to carry out a discharge in gaseous media, the RC-generators of pulses are commonly used (Fig. 2). A capacitor is charged using the current source through the ballast resistance $R$. As the electrodes TE (a vibrating TE is used in this method) and P (TE is the electrode-tool and $\mathrm{P}$ is the workpiece) move to contact, a breakdown of a gaseous gap occurs at a certain $l_{\min }$ length. Because of the polar effect, the transfer of the eroded material mostly from the anode onto cathode involves the formation of a site with certain physicochemical properties across the latter.

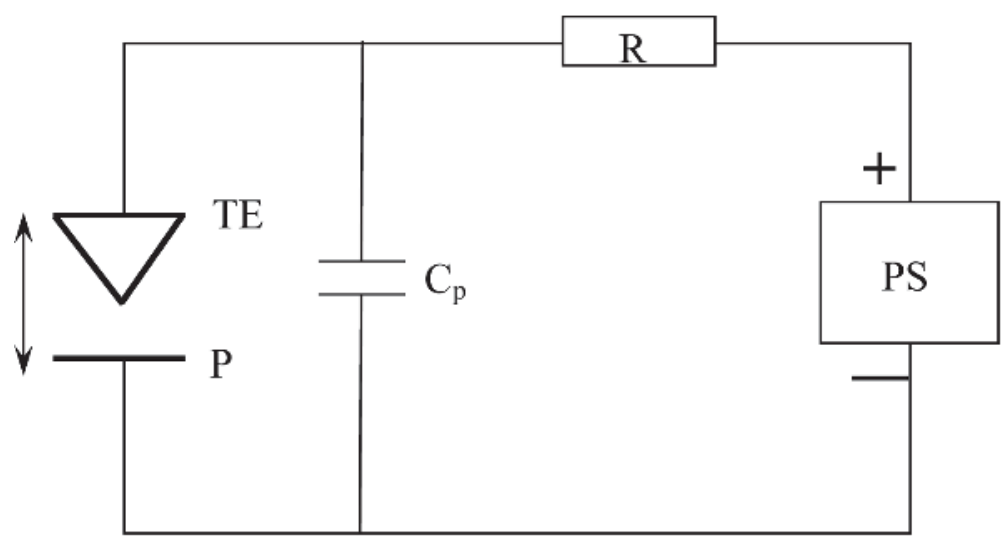

Fig. 2. Scheme of the simplest RC-generator.

As a rule, the surface layer of the cathode changes its composition and structure due to the ESD. The characteristics of this layer can be varied within the wide range due to a selection of the electrode material, composition of the interelectrode medium, parameters of the pulse discharges and other conditions when forming a layer on the cathode. It is obvious that the ESD ensures wide possibilities for creation of the working areas with specified operational characteristics.

The amount of the anode material that is transferred during a single act of the discharge is small. Thus, for a hard alloy (titanium-tungsten) at the discharge energy of $1 \mathrm{~J}$ it is $2-310^{-6}$ g. Therefore, in order to form a layer of a required thickness across the cathode, both a periodic commutation between the anode and cathode and a displacement (scanning) of the anode along the treated surface or a displacement of the cathode with respect to the fixed anode are necessary.

The periodic commutation of the cathode with anode is performed using various facilities, e.g., special vibrators, rotating disks or discs with the electrodes in the form of plates or small wires located along its perimeter which contact the cathode due to vibration, and the vertical feed of the automatic controller.

The ESD versions were developed to form the layer and perform the polar transfer using a powder material that was introduced into IEG. Here, the ESD advantages of using the compact electrodes are combined with the wide possibilities of the powder methods for the coating deposition. 


\subsection{Dynamics of transfer of the electrode materials at EDS}

In the case of the compact materials used as the anode, the most popular variant of treatment is one at which the commutation between the anode and cathode is possible due to vibration. The processes are performed at $U \sim 15--200 \mathrm{~V}$, the pulse duration is in the range from the tens of microseconds to milliseconds, the frequency of vibration is of 50-$300 \mathrm{~Hz}$, and the amplitude is up to $0.2-0,5 \mathrm{~mm}$. The breakdown of the interelectrode gap at the indicated voltages can occur at the distances that equal $\sim 0.01-10 \mu \mathrm{m}$. Taking the frequency and amplitude of vibration into account, the time of passing the indicated distances by the anode is from several to the tens of microseconds. Hence, the discharge can occur completely in the gaseous phase and it can stop upon the contact of the electrodes. At $\mathrm{U}<100 \mathrm{~V}$ the discharge develops and terminates actually upon the contact of the electrodes.

In $10^{-7}-10^{-8} \mathrm{~s}$ after the breakdown and the beginning of formation of a discharge channel (a plasma jet of the discharge), the evaporation from the surface of the electrodes in the form of jets and vapors and the ejection of the liquid phase by means of dispersion starts. Since these phenomena take place in a fairly small interelectrode gap that in addition decreases continuously, favorable conditions are created for the transfer of the flow of energy to the counter electrodes.

Upon the current pulse of certain duration, the electrodes manage to approach each other almost to a full contact before the discharge termination. But the full contact apparently fails to occur between the anode and cathode, since the pressure of the vapors of the metals in the evaporation zone can reach $10^{8} \mathrm{~Pa}$ which exceeds considerably the pressure that is developed by the electromagnetic system of the vibrator in the contact zone.

The liquid volumes of the approached anode and cathode are exposed to the effect of several forces: a hydrodynamic pressure of the flames, a gas-kinetic pressure of the discharge channel, and the electrodynamic force, etc. The volumes of the liquid metal are distorted under the total effect of those forces and eject from the dimple. Since this takes place during the contact, the integration of the liquid phases of the electrode materials occurs along with their convective mixing.

Due to the polar effect and the aforementioned factors, the quantity of the liquid phase across the anode must be substantially higher compared to that of the cathode and, hence, the surface layer that was formed on the cathode must consist mainly of the anode material. But the convective mixing is responsible for the fact that a fairly great amount of the material of the cathode is also distributed in this layer. In addition, it is noteworthy that the treatment takes place in a gaseous medium that comprises the elements which can form the chemical compounds (oxides and nitrides) that determine the surface layer.

\subsection{Effect of various conditions on the formation of a surface layer on the cathode}

The formation of the ESD surface layer is performed by a successive local exposure to the pulse discharge of all sites of the treated cathode. As a rule, the required characteristics of the layer can be achieved by a repeated travel of the anode over one site of the cathode. In most case, in order to obtain a uniform layer over the entire treated surface, a constant shift of the anode with respect to the zone of interaction of the discharge with the cathode is necessary. This shift is usually selected experimentally.

The quantity of the transferred material onto the cathode is generally fixed in the form of a change in the cathode weight. The weight change in the cathode during $1 \mathrm{~min}$ upon the 
treatment of $1 \mathrm{~cm}^{2}$ of the surface is generally referred to as a specific gain. It is actually a characteristic of the intensity of the ESD process. A detailed study of the formation of the layer across the cathode and the anode erosion under different conditions of treatment showed that the effect of the following factors is most significant, namely, that of the parameters of the pulse discharge, the duration of the treatment, the nature of the electrode materials, the interelectrode medium and a form of the anode motion with respect to the cathode.

The dynamics of the formation of the surface layers is characterized by the fact that the intensity of the anode material transferred to the cathode is found to be the highest at the initial moments of the process, but then it decreases. Eventually, the weight gain of the cathode may change for the inverse process, i.e., its erosion ("a negative gain"). The combination of these two processes, at a fairly high share of the latter, may lead to a restriction in the thickness of the coatings that is really observed in many cases. Usually, in the range of the discharge energies of $0.1-3 \mathrm{~J}$ the treatment of a $1 \mathrm{~cm}^{2}$ surface during $0.5-2$ min yields a maximum (or close to it) value of the cathode gain.

There are various ways to increase the rates of deposition and thicknesses of the deposited layers. One such is the use of the rotating electrode instead of the vibratiory one. In the latter case, a position of the discharge channel and a zone of interaction of the electrodes during the discharge, shift in the direction of motion of the anode and the erosion trace widens along the cathode surface. This leads to the change of the thermal mode of the treatment which, as a result, affects the obtained thickness of the coating. In this case, the thicknesses of the coatings may reach $1-2 \mathrm{~mm}$, which significantly exceeds the thicknesses developed under conditions of the vibratory electrode. However, the surface roughness may also increase.

The restriction of the thicknesses of the coatings results from the dynamics of changes in the values of the remaining strains in the manufactured coatings. The obtained results show that with an increase in the specific duration of the alloying, the level of the stretching remaining strains in the developed layers increases. However, definite values of a specific duration of the alloying exist for each kind of material at which the maximum level of the stretching remaining strains is observed.

Study of the effect of a composition of the gaseous environment showed that its change not only allows one to control the deposition rate, but also the composition and structure of the developed layers. This was manifested most vividly during the treatment in the reducing media (hydrogen and argon).

In the process of developing the surface layer on the cathode, between the liquid phases of the electrode material there occurs interaction which leads to establishing chemical bonds between the components and to the formation of intermetallic compounds and alloys, as well as the development of the process of self- and heterodiffusion.

Under these situations of the materials interaction the processes of crystallization, mass transfer and other phenomena occur under extremely nonequilibrium conditions that result in formation of nano- and fine-crystalline structures up to the formation of the amorphous structures.

\section{The ESD by the AI-Sn alloy electrode-tool}

\subsection{The ESD installation and the TE material}

The power for the ESD was supplied using an ALIER-31 installation (SCINTI, Moldova). The ALIER type electrospark power supplies are successfully used for various types of the 
electrospark deposition of coatings. A specific feature of this installation is that the frequency of the generated pulses is not directly related to the TE vibration frequency; it is set independently. Thus, it depends on the pulse energy. Table 1 lists the parameters of the technological pulses of the ALIER-31 generator.

In this study, we used all seven modes of the ALIER-31 installation (Table 1) at a constant treatment time of $1 \mathrm{~min}$; the frequency of the set pulses corresponded to that shown in Fig. 3. This was achieved by means of a special frequency regulator (energy coefficient). Here, it should be taken into account that, since we used an installation with a manual TE, the real number of pulses in a gap depended on the operator's hand and on the conditions in it. The number of pulses in a gap can be assumed to be $0.6-0.9$ from the values corresponding to those presented in Fig. 3.

As a TE, we used the rods from a specially prepared Al-Sn alloy doped with copper and titanium $(\sim 1 \mathrm{wt} \% \mathrm{Cu}$ and $0.1 \mathrm{wt} \% \mathrm{Ti})$ with a diameter of $\sim 8 \mathrm{~mm}$.

\begin{tabular}{|c|c|c|c|c|}
\hline No. & Mode & $\begin{array}{c}\text { Pulse duration, } \\
\mu s( \pm 10 \%)\end{array}$ & $\begin{array}{c}\text { Pulse current amplitude, } \\
\mathrm{A}( \pm 20 \%)\end{array}$ & Pulse energy, J \\
\hline 1 & 1 & 16 & 125 & 0.036 \\
\hline 2 & 2 & 31 & 125 & 0.07 \\
\hline 3 & 3 & 62 & 175 & 0.2 \\
\hline 4 & 4 & 125 & 175 & 0.39 \\
\hline 5 & 5 & 250 & 175 & 0.79 \\
\hline 6 & 6 & 500 & 175 & 1.58 \\
\hline 7 & 7 & 1000 & 175 & 3.15 \\
\hline
\end{tabular}

Table 1. Parameters of the technological pulses of the generator of the ALIER-31 installation

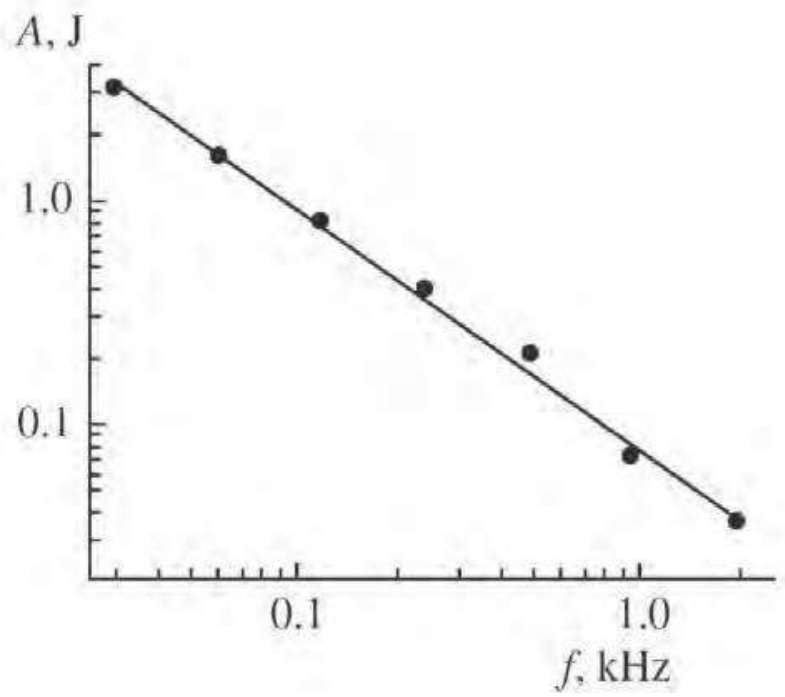

Fig. 3. Frequencies and values of the pulse energies in various modes of the electrodischarge treatment. 
The alloy of the required chemical composition was melted in a graphite melting pot in the inductor of a V4I10U high-frequency installation; then, it was poured into a specially prepared chill mould with a size of $8.50 \mathrm{~mm}$. The procedure of the manufacturing consisted of the following operations: (a) preparation of the working mixture; (b) melting in an induction furnace; (c) pouring the melt into a chill mold; and (d) topping, clearing, and turning.

In order to obtain an alloy with a preset composition, we used pure aluminum and tin. The doping components were introduced in the form of foundry alloys $(50 \% \mathrm{Al}+50 \% \mathrm{Cu}$ and $90 \% \mathrm{Al}+10 \% \mathrm{Ti})$. The working mixture was calculated with respect to the mean content of the elements: $20 \mathrm{wt} \% \mathrm{Sn}, 1 \mathrm{wt} \% \mathrm{Cu}, 0.1 \mathrm{wt} \% \mathrm{Ti}$, and the rest was $\mathrm{Al}$. As a sample, we used a D1 aluminum alloy (State Standard GOST 4784). Manual treatment was carried out. The TE and the sample were weighed before and after the treatment in each experiment. Their surface (before and after the treatment) was studied by means of a scanning electron microscopy (a TESCAN scanning electron microscope with an INCA Energy EDX detachable device for the element analysis of the surface (Oxford, Great Britain)).

\subsection{Composition and structure of the TE applied}

Figure 4 shows a diagram of the state for the Al-Sn binary system. One can see that at room temperature (up to the tin melting point of $228^{\circ} \mathrm{C}$ ), the material used as a TE (AlSn20) must be an aluminum matrix with tin metal dispersed in it. This is confirmed by the results of the scanning electron microscopy and the EDX element analysis (Fig. 5), as well as by the sample surface scanning with the simultaneous determination of the aluminum and tin (Fig. 6). One can see that the TE is really an aluminum matrix with tin particles with a size of $3-5$ $\mu \mathrm{m}$ dispersed in it (Fig. 6).

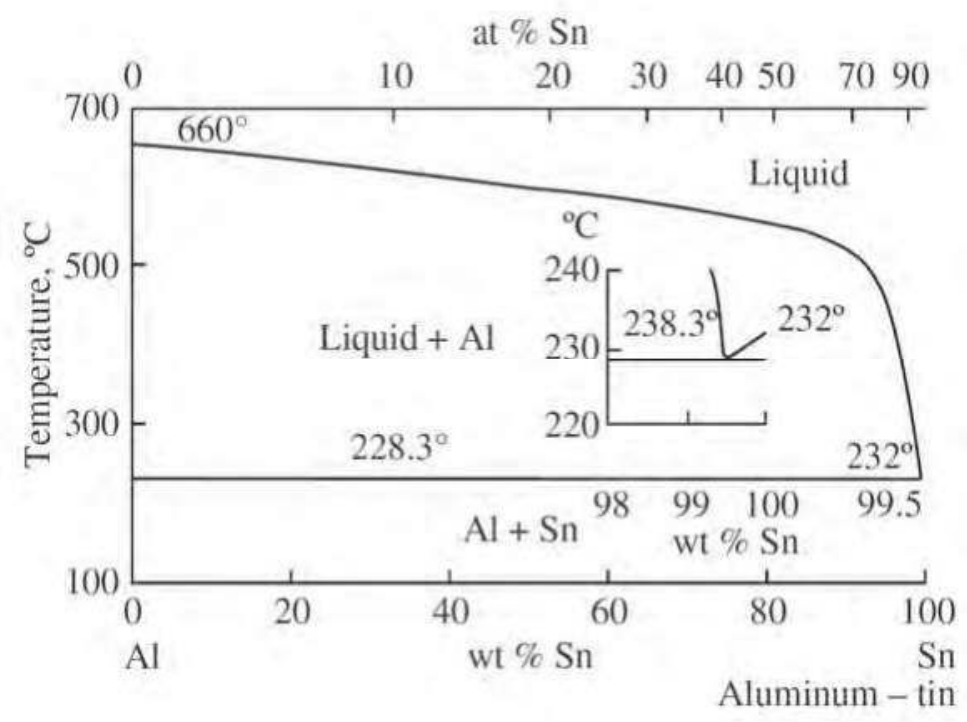

Fig. 4. State diagram for the aluminum-tin system. 
(a)
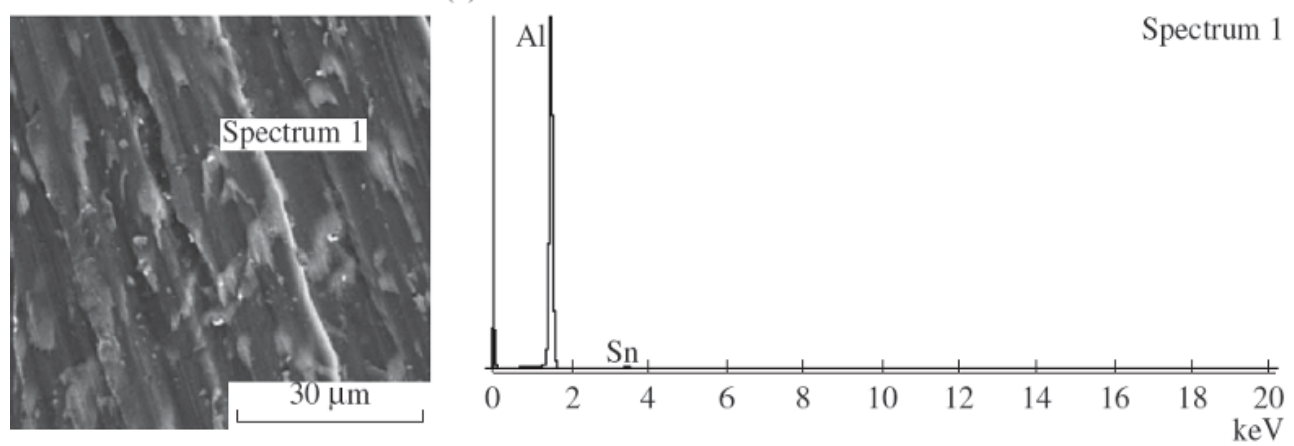

(b)
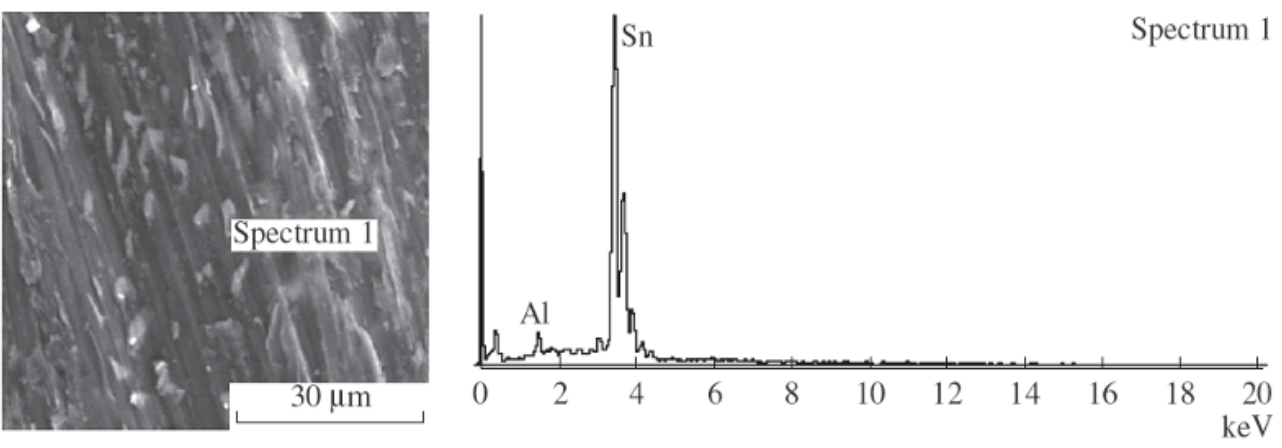

Fig. 5. SEM images of the TE surface and the EDX spectra of the matrix (a) and the dispersed tin (b). The crosses in the microphotographs show the places of the local EDX analysis.

\subsection{Mass transfer effects}

Figure 7 shows changes in the weight in the process of the experiments both for the sample and for the TE. It is seen that, at relatively low pulse energies (and, respectively, high frequencies, see Fig. 3), a loss in the sample mass occurs, which increases as the pulse energy grows. This is region I in Fig.7. In this region, a loss in the TE mass is also observed; however, it is relatively small. It is obvious that region I is the region characteristic of electroerosion treatment in an air medium, which is characterized by the removal of substance from the work piece surface (that increases with the growing pulse energy) and by the low wear of the TE. As the pulse energy increased, the situation varied significantly. At relatively high energies, a polar transfer on the sample surface was observed; it was accompanied by weight gain. The transfer coefficient (the ratio of the gained weight to the weight of the substance removed from the TE) was $\sim 0.3$. This region (II in Fig. 7 ) is the ESD classical variant with respect to the TE material used. In the intermediate region, considerable wear of the TE was observed at relatively low material transfer on the sample surface (Fig. 7). 


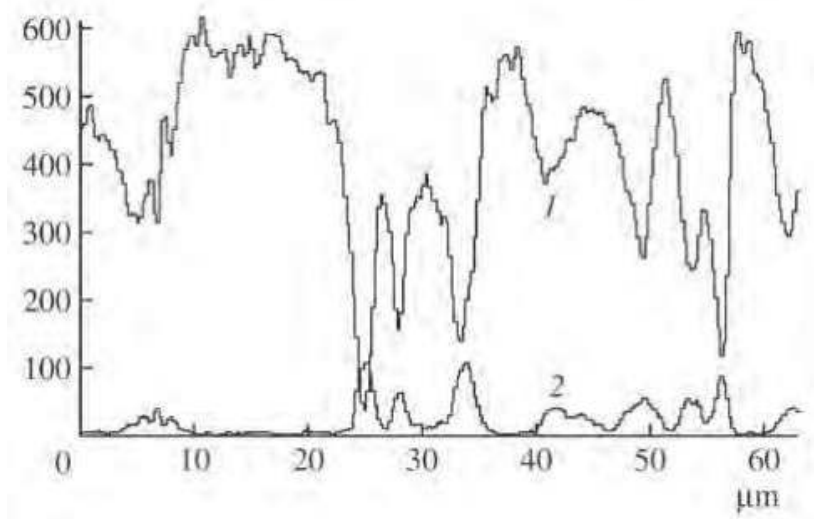

Fig. 6. Distribution of the aluminum (1) and tin (2) over the TE surface. The levels of the EDX spectrum are given in relative units.

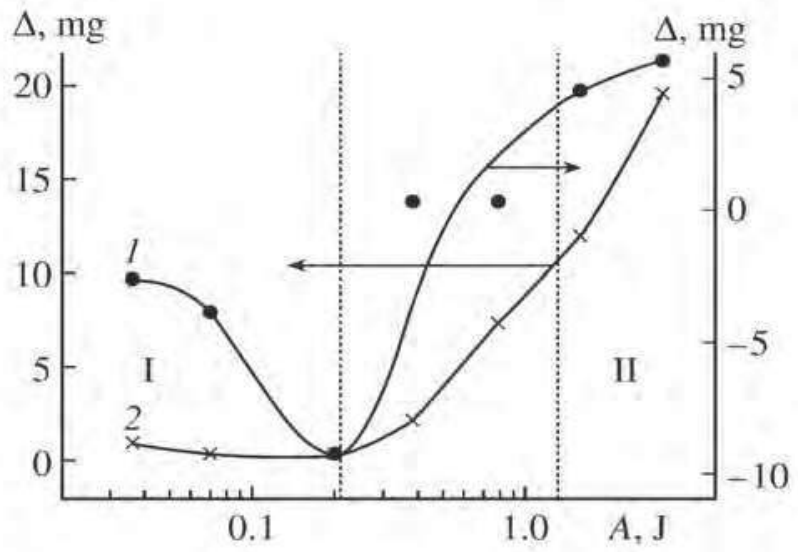

Fig. 7. Influence of the pulse energy on the variation of the weight of the sample (1) and the TE (2).

\subsection{Morphology and composition of the surface}

Figure 8 shows the surfaces of the sample and the TE after treatment in the modes corresponding to region I in Fig. 7 and to the intermediate region. It is clear from the results of element analysis (Table 2) that they are a mixture of oxides and nitrides of aluminum (the sample) as well as of oxides of aluminum, tin, and copper (the TE). The presented photographs of the surface and the analysis results are typical for treatment in modes 1-5. Here, the composition of the surfaces could change insignificantly. In particular, on the sample surfaces in modes $2-5$, there was tin (a trace amount); sometimes, copper. The composition of the TE and the sample comprised carbon. This can be caused by the fact that the TE melting was performed in a graphite melting pot. The morphology of the surfaces obtained in these conditions is characteristic of the fritted surfaces. 


\begin{tabular}{|c|c|c|c|c|c|}
\hline Element & $\mathrm{Al}$ & $\mathrm{Sn}$ & $\mathrm{O}$ & $\mathrm{N}$ & $\mathrm{C}$ \\
\hline wt \% & $50.0 \pm 4.8$ & $34.8 \pm 2.6$ & $7.7 \pm 1.5$ & $2.2 \pm 0.6$ & $5.3 \pm 2.6$ \\
\hline
\end{tabular}

Table 2. Composition of the sample's surfaces after treatment in modes 6 and 7 (mean values)

\begin{tabular}{|c|c|c|c|c|c|}
\hline Element & $\mathrm{Al}$ & $\mathrm{Sn}$ & $\mathrm{O}$ & $\mathrm{N}$ & $\mathrm{C}$ \\
\hline wt $\%$ & 19.1 & 61.5 & 11.5 & 4.9 & 3.0 \\
\hline
\end{tabular}

Table 3. Element composition of the wire on the TE after treatment in mode 6 (see Fig. 9a)

The given results confirm the results of the measurements of the loss in mass; it follows from the latter that region $\mathrm{I}$ is the region of the electroerosion treatment in an air medium. A similar situation is observed for the samples treated in modes 4 and 5 (see Table 1, the region of intermediate modes shown in Fig. 7).

As to region II in Fig.7, alongside with the composition and morphology of the surface characteristic of region I and the intermediate region, on the surfaces of the samples and the TE treated in these modes, specific structures in the form of wires with a diameter less than $1 \mu \mathrm{m}$ are registered (Fig. 9). Figure 10 shows nanowires at higher magnification. It is seen that their diameter ranges within $\sim 200-600 \mathrm{~nm}$. Table 3 presents the composition of the samples surface after treatment in these conditions. One can see that, in comparison with the other modes, the tin concentration on the surfaces of the samples sharply increases (Fig. 11). The analysis of the wire's composition shows that this is tin for the most part (Table 3, Fig. 9a). Table 3 gives the results of the element analysis of a large fragment of the wire on the $\mathrm{TE}$; it follows from the data that the tin concentration in the wires is more than $60 \mathrm{wt} \%$ (also see the EDX spectrum in Fig.9a).

However, it is impossible to answer the question whether they consist exclusively of tin (its oxides) or comprise aluminum and its oxides as well (yet in a less amount than tin), because the analysis technique applied records the ratio of the components in volumes that exceed the volume of only the wires, and it partially includes the volumes of the surface layers on which these wires locate.
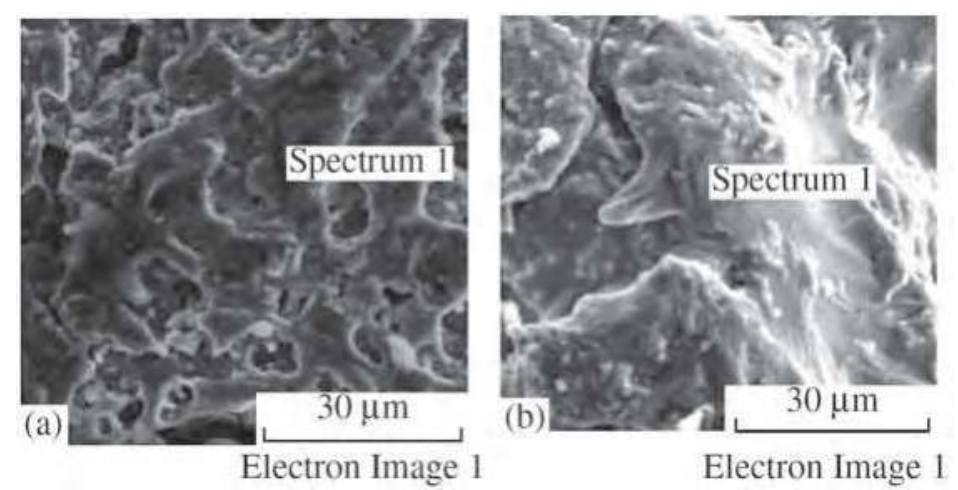

Fig. 8. Morphology of the surface of the sample (a) and the TE (b) after treatment in modes 1 (a) and 4 (b). 
(a)
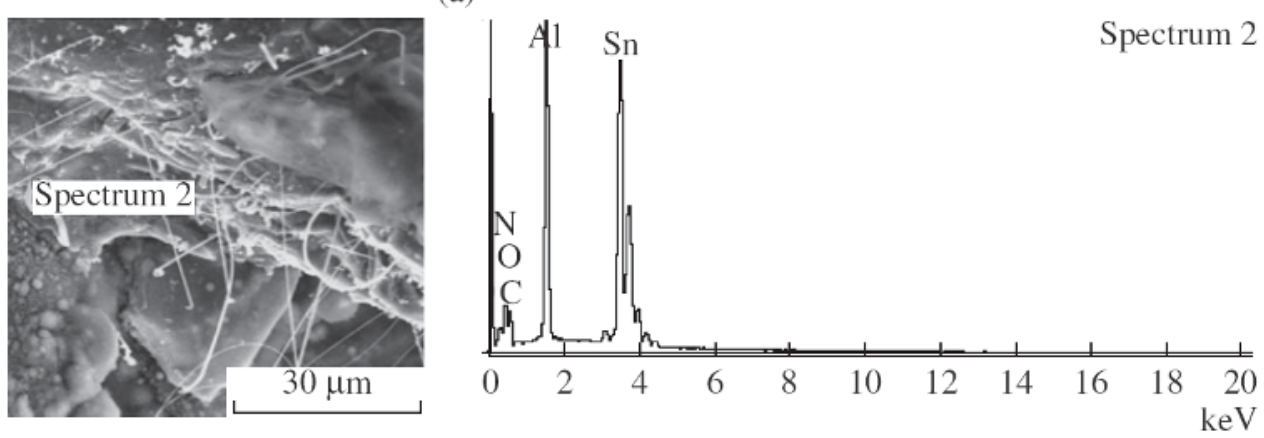

(b)
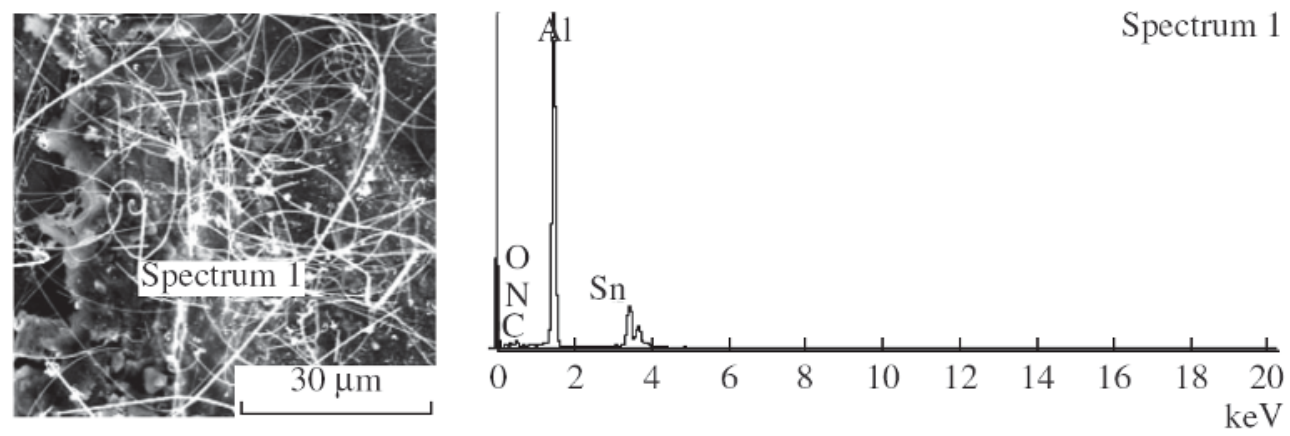

Fig. 9. Morphology of the surface and the EDX spectra of the TE (a) and the sample (b) after treatment in modes 6 (a) and 7 (b).

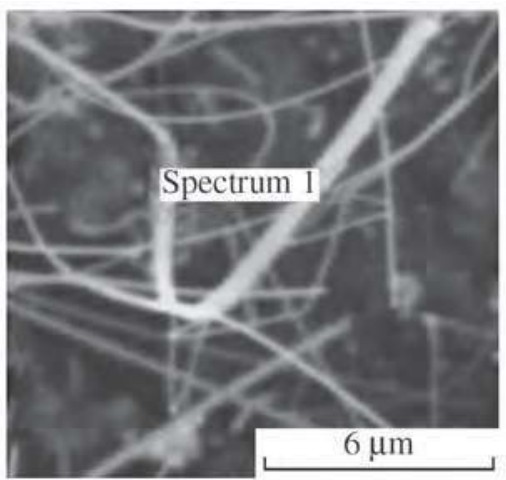

Fig. 10. Surface of the sample after treatment in mode 7 (fragment of Fig. 9b). 


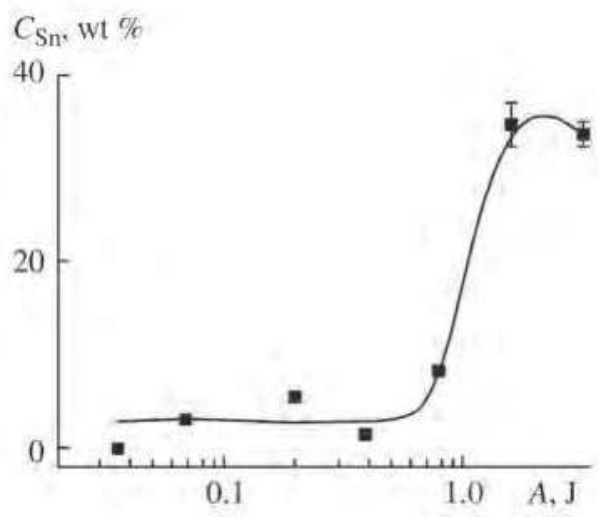

Fig. 11. Influence of the pulse energy on the tin concentration on the sample surfaces.

\subsection{Physical bases of obtaining the nanowires.}

The cause for the formation of the nanowires is apparently the specific character of the Al$\mathrm{Sn}$ state diagram at temperatures that are higher than the tin melting point $\left(228^{\circ} \mathrm{C}\right)$ but lower than the melting point of the Al-Sn alloy ( $\sim 655^{\circ} \mathrm{C}$ for the AlSn20 alloy) (Fig. 4). In this case, the system represents melted particles of dispersed tin being in a solid matrix of aluminum (Fig. 4 -6). The transfer of these particles to the interelectrode gap occurs due to the ponderomotive forces that deform the surface of a melted drop if the surface tension force of the melt-air system is sufficiently low for the melted particles. It is known, that minimal values of the surface tension forces are observed precisely for the tin melt-air system. It is obvious that, since the surface tension forces of the melted particles of tin (or tin partially enriched in aluminum and their oxides are low, the ponderomotive forces, which appear due to the presence of a field in the gap, exceed them so that, as a result, wires with a diameter of $\sim 1 \mu \mathrm{m}$ and less are formed (Fig 9, 10).

The effects of this kind must be observed not only for the Al-Sn systems but also for any other systems that, at certain temperatures, are a system of melted particles in a solid matrix, for example, the Al-Pb system.

As to the possible practical applications that follow from the results of the experiments that have been described, we should mention the possibility of increasing the concentration of a low-melting component in the near-surface layer under the ESD conditions with the use of a TE of this kind (Fig. 11).

\section{Mechanical properties of the surfaces obtained after the ESD by the AI-Sn alloy}

\subsection{Wear resistance of the coatings after the ESD by the Al-Sn alloy}

The study of the mechanical properties of the surfaces developed with the formation of nanowires from the easily melted component was carried out using a see-saw friction machine (Institute of the Applied Physics, Moldova Academy of Sciences). The rectangular $3 \times 5 \times 55-\mathrm{mm}$ plates from aluminum alloy D1 (State Standard GOST 4784 ) were used as the specimens to be studied (the surface with a smaller size contacted a counterbody). The counterbody was a rectangular $3 \times 25 \times 30-\mathrm{mm}$ plate from the hardened steel St 45 with 
microhardness of $650 \pm 50 \mathrm{kgf} / \mathrm{mm}^{2}$. The surface of a smaller size contacted with the surface under study, thus, the area of a contact was $9 \mathrm{~mm}^{2}$ and the counterbody was fixed at an angle of $90^{\circ}$ with respect to the exposed specimens.

The counterbody performed a reciprocable movement relatively the specimen under study at a rate of 45 double movements a minute. The length of the working surface that contacted the conterbody was $48 \mathrm{~mm}$. The initial microhardness of the unfinished surface of the alloy D1 was about $100 \mathrm{kgf} / \mathrm{mm}^{2}$. A vaseline oil was used during the testing friction process.

The testing comprised two stages, since, as a rule, the surfaces with high roughness result from the ESD. At the first stage during $10 \mathrm{~h}$ the surface under study and the counterbody were exposed to a preliminary wear-in. It consisted in a successive increase in the load from 20 to $100 \mathrm{kgf} / \mathrm{cm}^{2}$; at the beginning of the work it was every $1 \mathrm{~h}$ of work and then every $2 \mathrm{~h}$ of work. At the second stage, the major testing process took place; it was performed for $20 \mathrm{~h}$ at a load of $100 \mathrm{kgf} / \mathrm{cm}^{2}$.

Prior to the testing, the roughness of the specimen under study was measured after the ESD $\left(R_{a}{ }^{0}\right)$. Similar measurements were taken after the stage of the preliminary wear-in $\left(R_{a}\right)$, as well as after the termination of the testing $\left(R_{a}\right.$ II). The weight loss measurements were performed both for the counterbody $(\Delta \mathrm{Ucb})$ and for the tested specimens $(\Delta \mathrm{U})$. The latter was a sum of losses after the preliminary wear-in and after the main tests at a fixed load. A degree of the wear was estimated both in the absolute $\left(\Delta \mathrm{U}, \Delta \mathrm{Ucb}^{\mathrm{cb}}\right)$ and in the relative $(\Delta \mathrm{Ucb} / \Delta \mathrm{U})$ values.

The ESD of the tested specimens was carried out using an ALIER-31 installation (SCINTI, Moldova) in the modes of 4 and 6. A wear degree was estimated compared both to the unfinished and the treated surfaces, but with the (aluminum) electrode which lacked the easily melted component (Sn). The use of the aluminum electrode as the TE, appeared to be possible (provided the treated specimens gained in weight) only in mode 4 (in more intense modes, the welding of the TE to the specimen was registered and the EDS was impossible). Therefore, the results of comparison of AlSn20 used as the TE both in mode 4 and mode 6 are listed in Table 4).

\begin{tabular}{|c|c|c|c|c|c|c|c|c|}
\hline № & $\begin{array}{c}\text { Material } \\
\text { TE/P }\end{array}$ & Mode & $\mathrm{R}_{\mathrm{a}}{ }^{0}, \mu \mathrm{m}$ & $\mathrm{R}_{\mathrm{a}} \mathrm{I}, \mu \mathrm{m}$ & $\mathrm{R}_{\mathrm{a}}{ }^{\mathrm{II}}, \mu \mathrm{m}$ & $\Delta \mathrm{U}, \mathrm{mg}$ & $\begin{array}{c}\Delta \mathrm{Ucb}^{\mathrm{c}}, \\
\mathrm{mg}\end{array}$ & $\begin{array}{l}\Delta \mathrm{U}^{\mathrm{cb}} \\
/ \Delta \mathrm{U}\end{array}$ \\
\hline 1 & $-/ \mathrm{Al}$ & - & $0.72 \pm 0.08$ & $0.32 \pm 0.21$ & $0.25 \pm 0.15$ & 137.3 & 0.4 & \multirow{2}{*}{$2 \cdot 10^{-2}$} \\
\hline 2 & $-/ \mathrm{Al}$ & - & $0.52 \pm 0.25$ & $0.17 \pm 0.08$ & $0.20 \pm 0.10$ & 114.3 & 4.3 & \\
\hline 3 & $\mathrm{Al} / \mathrm{Al}$ & 4 & $26.0 \pm 4.6$ & $11.0 \pm 1.7$ & $7.6 \pm 1.7$ & 22.4 & 1.5 & $7 \cdot 10^{-2}$ \\
\hline 4 & $\mathrm{Al}-\mathrm{Sn} / \mathrm{Al}$ & 4 & $13.1 \pm 2.4$ & $6.5 \pm 0.9$ & $5.9 \pm 1.0$ & 12.1 & 9.35 & 0.8 \\
\hline 5 & $\mathrm{Al}-\mathrm{Sn} / \mathrm{Al}$ & 4 & $14.2 \pm 2.6$ & $7.5 \pm 2.0$ & $7.8 \pm 1.3$ & 2.6 & 5.95 & 2.3 \\
\hline 6 & $\mathrm{Al}-\mathrm{Sn} / \mathrm{Al}$ & 4 & $13.1 \pm 1.2$ & $7.6 \pm 1.6$ & $7.8 \pm 1.0$ & 5.1 & 5.95 & 1.2 \\
\hline 7 & $\mathrm{Al}-\mathrm{Sn} / \mathrm{Al}$ & 4 & $10.7 \pm 1.6$ & $8.5 \pm 1.3$ & $8.8 \pm 1.0$ & 0.6 & 6.7 & 11.2 \\
\hline 8 & $\mathrm{Al}-\mathrm{Sn} / \mathrm{Al}$ & 4 & $15.1 \pm 3.4$ & $8.6 \pm 1.0$ & $5.8 \pm 1.1$ & 7.6 & 32.0 & 4.2 \\
\hline 9 & $\mathrm{Al}-\mathrm{Sn} / \mathrm{Al}$ & 4 & $18.9 \pm 1.5$ & $10.9 \pm 1.5$ & $7.5 \pm 1.5$ & 9.1 & 37.0 & 4.1 \\
\hline 10 & $\mathrm{Al}-\mathrm{Sn} / \mathrm{Al}$ & 6 & $17.9 \pm 1.5$ & $12.9 \pm 2.2$ & $12.1 \pm 2.0$ & 4.1 & 50.8 & 12.4 \\
\hline 11 & Al-Sn/Steel & 4 & $5.90 \pm 0.85$ & $2.92 \pm 0.79$ & $2.62 \pm 0.61$ & 3.3 & 2.85 & 0.9 \\
\hline 12 & $\mathrm{Al}-\mathrm{Sn} / \mathrm{Al}$ & 6 & $10.7 \pm 2.4$ & $9.4 \pm 3.2$ & $10.5 \pm 2.6$ & 0.3 & 1.03 & 3.4 \\
\hline 13 & $\mathrm{Al}-\mathrm{Sn} / \mathrm{Al}$ & 6 & $7.3 \pm 1.2$ & $7.3 \pm 1.5$ & $7.0 \pm 1.0$ & 0.25 & 0.9 & 3.6 \\
\hline
\end{tabular}

Table 4 . Results of the wear tests of the surfaces 
The ESD process was performed manually. As was aforementioned. the ESD indices depend strongly on the method for the coating deposition. Two methods were used in the considered experiments. The first experiment consisted in the successive coating of the entire treated surface during $4 \mathrm{~min}$.. i.e.. first. one part. then another. etc. In the second case. the whole surface was coated during $1 \mathrm{~min}$ and then the following treatment was performed. The overall time of treatment was $4 \mathrm{~min}$.

The surface roughness after the ESD in the first variant of treatment was significantly lower $\left(R_{a}=12.8 \pm 1.3 \mu \mathrm{m}\right)$. In the second case it was $17.3 \pm 2.0 \mu \mathrm{m}$. and during the treatment using the Al TE it was $26.0 \pm 4.5 \mu \mathrm{m}$ (test 3. Table 4).

The EDS was performed not only on the specimens from the aluminum alloys D1. but also on steel St 45. In this case the roughness was considerably lower $\left(R_{a}=5.90 \pm 0.85 \mu \mathrm{m}\right.$ (test 11. Table 4).

In the process of the preliminary wear-in. $R_{a}$ decreased and. as a rule. the changes in the roughness in the process of the testing were not registered (Table 4). In certain experiments. during the treatment in mode 6. i.e.. under the conditions when the maximum roughness of the treated surface was observed. the latter was exposed to a mechanical treatment (polishing) before the testing. About a $0.2 \mathrm{~mm}$-alloyed layer was removed (tests 12. 13. Table 4). In this case the roughness of the surface remained unchanged both in the preliminary wear-in and the main processes.

The results of Table 4 show that unlike the unfinished surface and the surface treated only by $\mathrm{Al}$ for which $\Delta \mathrm{Ucb} / \Delta \mathrm{U} \ll<1 . \Delta \mathrm{Ucb} / \Delta \mathrm{U}>1$ resulted from the treatment by the AlSn20 alloy and only for the surfaces developed in mode 6 (i.e.. according to the results with an increased Sn concentration in a coating presented in part 4). the wear of the counterbody is higher by more than an order of magnitude than that of the tested surface (Table 5. Figs. 12. 13).

Even after the mechanical removal of a part of the treated surface (tests 12. 13. Table 4) this value is $3.5 \pm 0.1$. i.e.. the counterbody wear exceeds the wear of the tested surface $(0.25--0.3$ mg during the entire testing time) by a few times.

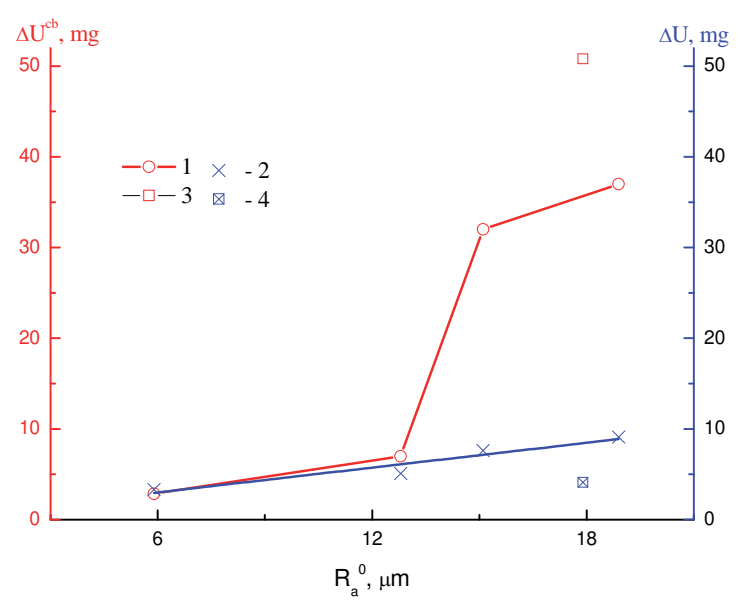

Fig. 12. Dependence of the absolute wear of the counterbody (1) and the treated surface (2) on the surface roughness. developed after the ESD in modes 4 and 6 ( 3 is the counerbody wear. 4 is the wear of the treated surface). 
It is evident that the wear of both the counterbody and that of the treated surface depend on its roughness (Fig. 12). However. upon the treatment by the Al electrode (without Sn) even in the presence of a considerably greater initial roughness of the specimen surface (prior to the tests) (test 3. Table 4) the opposite picture is observed. according to which the wear of the tested surface exceeds that of the counterbody by more than an order of magnitude (Fig. 12). It is clear that the only reason for the increased wear resistance of the ESD treated surfaces is the Sn-dopant introduced into their composition and which under the indicated conditions passes onto the treated surface also in the form of nanowires.

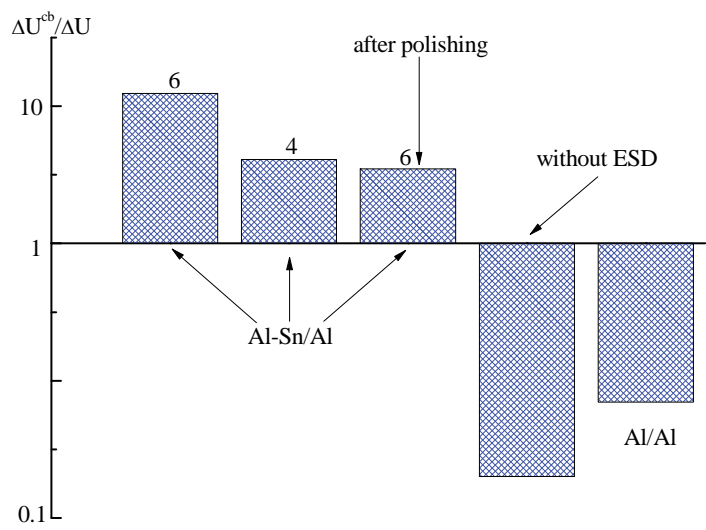

Fig. 13. Relative wear of the surfaces.

\subsection{On the causes of the increase in wear resistance of the surfaces treated by the Al- Sn alloy}

It seems evident that the increase in the wear resistance of the surfaces that have been treated by the Al-Sn alloy results precisely from tin presence in the TE. since the ESD using pure $\mathrm{Al}$ fails to lead to such effects. However. the Sn hardness is very low and the enhanced wear resistance can occur only due to the formation of certain compounds of $\mathrm{Sn}$ (or $\mathrm{Al}$ ) in the surface layer. The appropriate oxides $\left(\mathrm{Al}_{2} \mathrm{O}_{3}\right.$ and $\left.\mathrm{SnO}_{2}\right)$ are characterized by a considerably higher hardness. However. the element analysis of the coatings prior to and after the wear tests showed that the oxygen content in the surface layer (in at. \%) is considerably lower than a total amount of $\mathrm{Al}$ and $\mathrm{Sn}$ (on the average it was $\sim 12--20$ at. \% of oxygen. while the amount of $\mathrm{Al}$ and $\mathrm{Sn}$ is always above 50 at. \%). This is indicative of the fact that. if the above oxides are present. their concentration is relatively low.

Special XRD measurements. prior to and after the tests for wear showed that the coatings are amorphous (or the concentration of the crystalline formations in the coatings was out of the bounds of the XRD measurements). In other words. no relevant oxides. carbides. or nitrides. etc.. were found in the coatings.

Figure 14 shows the surface morphology and the EDX-spectrum of the surface after the wear tests (test 12. Table 4). A peculiarity of this test was that after the ESD the surface (prior to the wear tests) was exposed to a mechanic polishing with an $\sim 200 \mu \mathrm{m}$-thick layer being removed. This has led to an abrupt decrease in an absolute wear. while a relative wear of the counterbody also increased by several times compared to the surface wear that was subjected to the ESD (Table 4). 

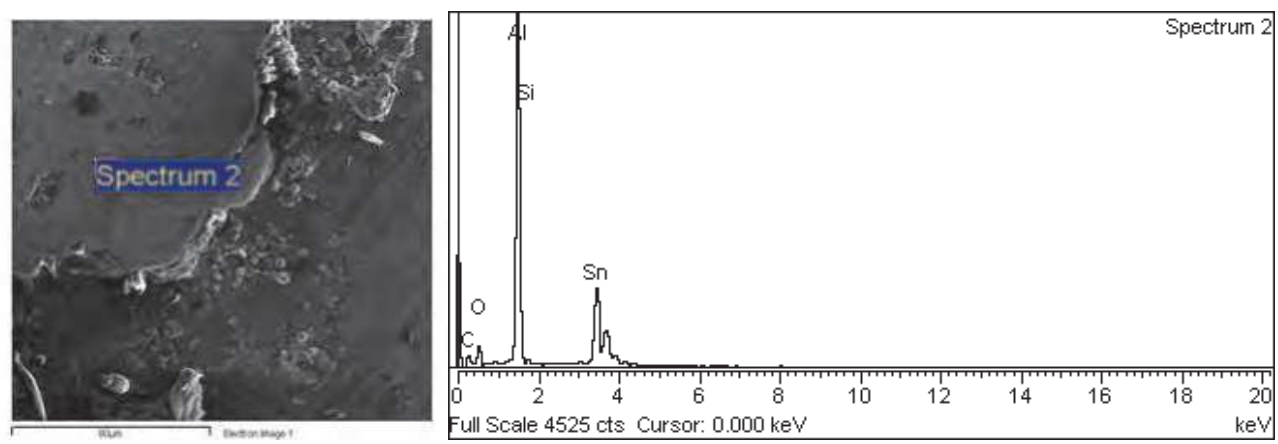

Fig. 14. SEM images of the specimen surface after the treatment and testing (test 12). On the right-hand side the spectrum of the EDX analysis is shown at a point that corresponds to the upper left-hand corner of Spectrum 2.

As is seen from the results in Figs. 14 and 15. in the area of the contact with the counterbody (where the element composition was measured) (Fig. 14). the Sn content is fairly high (wt $\%$ ): $\mathrm{Al}$ is $39.2 \mathrm{Sn}$ is 36.9 . and $\mathrm{O}$ is 12.8 . The test results showed that the counterbody wear (with no changes in the roughness both after the preliminary wear and fundamental tests) exceeded by 3.5 times the wear of the coating (Table 4 ).

It is of special interest that a classical method could be used to measure microhardness in the contact areas (both after the polishing and testing). It was similar to the microhardness of substrate $\left(\sim 100 \mathrm{kgf} / \mathrm{mm}^{2}\right)$. In other words. it seems evident that the only cause for the wear resistance increase in the coating compared to the counterbody is the presence of the $\mathrm{Sn}\left(\right.$ or $\left.\mathrm{SnO}_{2}\right)$ particles in it with such a degree of dispersion which fails to be registered by the classical XRD method. The most probable cause is the formation of the $\mathrm{SnO}_{2}$ (or some other tin compounds) nanowires in the coating. Since they cannot form a uniform coating. their local effect facilitates the counterbody wear. but they cannot be detected simultaneously using the classical identification methods.
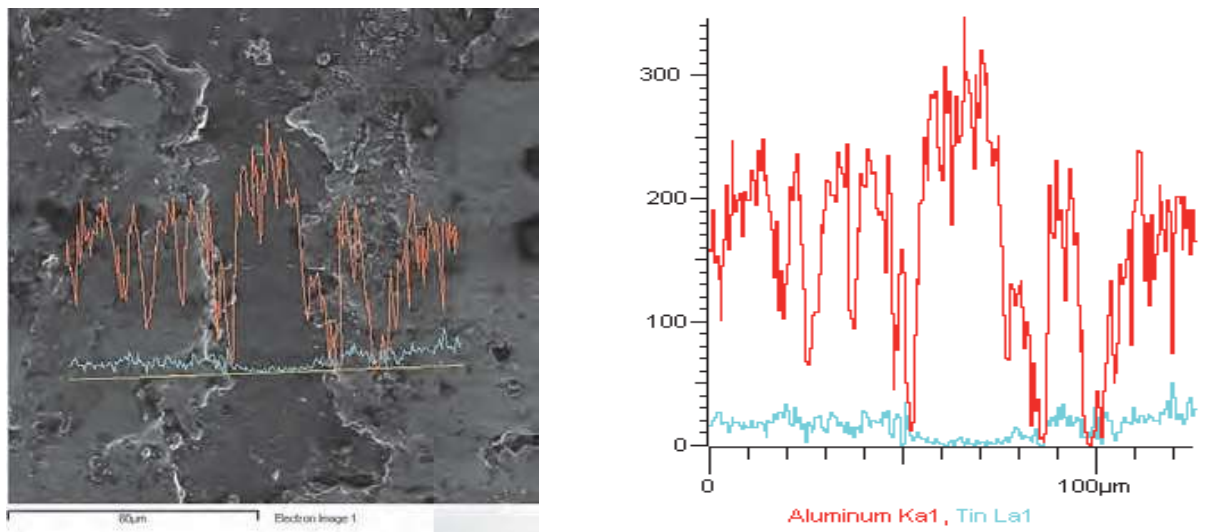

Fig. 15. Distribution of aluminum and tin on the treated and tested (test 12) surface. The EDX results are presented in the arbitrary units. On the left-hand side are the SEM images of the analyzed surface. 


\section{Conclusions}

The presented results have shown that the conditions of the electrodischarge treatment using the tool-electrodes that represent a mechanical mixture of an easily melting component in the matrix from a component with higher temperature of melting. facilitate the formation of nanowires from the compounds of an easily melting component (including the element of the environment). It is obvious. that these possibilities do not confine only to describing the ESD using the Al-Sn electrode. The method under consideration for obtaining the nanowires may be further developed both in the direction of a research of new double systems for the manufacturing of electrodes and in the direction of the work under conditions of the controlled medium where the electrical discharge occurs.

This work shows that the functional properties of the coatings obtained using the above method can have an extensive practical use. It is clear that not only the mechanical properties of these coatings may be of interest. The research in this direction is at the initial stage and the spheres of efficient application for such coatings have yet to be studied.

\section{Acknowledgements}

This study was performed under the financial support of the Moldova Academy of Sciences (the project: Electrophysicochemical Methods of New Materials and Coatings with Improved Functional Properties Manufacturing and Treatment) and Shevchenko Pridnestrov'e State University.

The author thanks V.I. Yurchenko and V.M. Fomichev for the assistance in performing the EDS process. and also V.I. Agafii and V.I. Petrenko for carrying out the mechanical tests.

\section{References}

Lazarenko. B.R.. Lazarenko N.I. (1964) Electrospark Machining of Metals. B.R. Lazarenko (Ed.). Consulting Bureau. New York. USA.

DiBitonto. D.D.. Eubank. P.T.. Patel. M.R.. Barruffet. M.A. (1989) Theoretical Models of Electrical Discharge Machining Process. I. A Simple Cathode Erosion Model. J. Appl. Phys. Vol. 66. No 9 pp. 4095-4103; Patel. M..R.. Barruffet. M.A.. Eubank. P.T.. II. The Anode Erosion Model. pp. 4104-4111.

Samsonov. G.V.. Verchoturov. A.D.. Bovkun. G.A.. Sitchev. V.S. (1976) Spark Discharge Doping. Nauka. Kiev. USSR.

Gitlevich. A.E.. Mihailov. V.V.. Parkansky. N.Y.. Revuzky. V.N. (1985) Electric Spark Alloying of Metal Surfaces. Stiintsa. Kishinev. USSR

Parkansky. N.Y.. Boxman. R.L.. Goldsmith. S. (1993) Development and Application of Pulsed-Air-Arc Deposition. Surf. Coat. Techn. Vol. 6. pp. 268-273.

Yurchenko. V.I.. Yurchenko. E.V.. Fomichev. V.M.. Baranov. S.A.. Dikusar. A.I. (2009) Obtaining of Nanowires in Conditions of Electrodischarge Treatment with Al-Sn Alloy. Surf. Engineering Appl. Electrochem. Vol. 45. No 4. pp.259-264.

Vol. A.E. (1959) Structure and Properties of Binary Metal Systems. N.V.Ageev (Ed.) Vol. 1. Fizmatgiz. Moscow. USSR. 


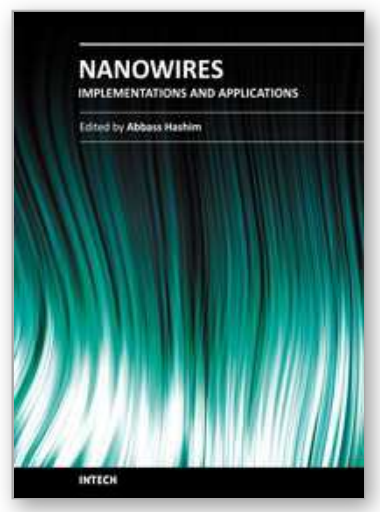

\author{
Nanowires - Implementations and Applications \\ Edited by Dr. Abbass Hashim
}

ISBN 978-953-307-318-7

Hard cover, 538 pages

Publisher InTech

Published online 18, July, 2011

Published in print edition July, 2011

This potentially unique work offers various approaches on the implementation of nanowires. As it is widely known, nanotechnology presents the control of matter at the nanoscale and nanodimensions within few nanometers, whereas this exclusive phenomenon enables us to determine novel applications. This book presents an overview of recent and current nanowire application and implementation research worldwide. We examine methods of nanowire synthesis, types of materials used, and applications associated with nanowire research. Wide surveys of global activities in nanowire research are presented, as well.

\title{
How to reference
}

In order to correctly reference this scholarly work, feel free to copy and paste the following:

Dikusar Alexandr (2011). Obtaining of Nanowires under Conditions of Electrodischarge Treatment, Nanowires - Implementations and Applications, Dr. Abbass Hashim (Ed.), ISBN: 978-953-307-318-7, InTech, Available from: http://www.intechopen.com/books/nanowires-implementations-and-applications/obtaining-of-nanowiresunder-conditions-of-electrodischarge-treatment

\section{INTECH}

open science | open minds

\section{InTech Europe}

University Campus STeP Ri

Slavka Krautzeka 83/A

51000 Rijeka, Croatia

Phone: +385 (51) 770447

Fax: +385 (51) 686166

www.intechopen.com

\section{InTech China}

Unit 405, Office Block, Hotel Equatorial Shanghai

No.65, Yan An Road (West), Shanghai, 200040, China

中国上海市延安西路 65 号上海国际贵都大饭店办公楼 405 单元

Phone: +86-21-62489820

Fax: +86-21-62489821 
(C) 2011 The Author(s). Licensee IntechOpen. This chapter is distributed under the terms of the Creative Commons Attribution-NonCommercialShareAlike-3.0 License, which permits use, distribution and reproduction for non-commercial purposes, provided the original is properly cited and derivative works building on this content are distributed under the same license. 